\title{
An Approximated Four-Phase Fuzzy Learning Algorithm
}

\section{Author: Shin-Jye Lee ${ }^{1}$; Ching-Hsun Tseng ${ }^{2}$}

\author{
Affiliation: Institute of Management of Technology, National Chiao Tung University, Taiwan ${ }^{1}$; \\ Department of Computer Science, University of Manchester, United Kingdom ${ }^{2}$ \\ E-mail: camhero@gmail.com ${ }^{1}$; hank131415go61@gmail.com $^{2}$
}

\section{DOI: 10.26821/IJSHRE.9.6.2021.9617}

\begin{abstract}
This work mainly aims to develop a dynamic modular fuzzy inference system by integrating the innovation of pattern recognition, the concept of fuzzy learning algorithm, the method of function approximation, the skill of system optimisation, and certain supports of multi-disciplines. With the development of the advanced fuzzy inference system, more and more methods have been proposed to effectively design the automatic fuzzy inference systems.
\end{abstract}

\section{Keywords: Fuzzy Inference Systems}

\section{INTRODUCTION}

This work mainly aims to develop a dynamic modular fuzzy inference system by integrating the innovation of pattern recognition, the concept of fuzzy learning algorithms, the method of function approximation, the skill of system optimisation, and certain supports of multi-disciplines. With the development of the advanced fuzzy inference system, more and more methods have been proposed to effectively design the automatic fuzzy inference systems. In order to construct the fuzzy membership function and rules automatically, all these methods can be considered as classification and clustering algorithm, partition refinement and rule generation technique innovating certain concepts and methods about function approximation. Meanwhile, a predetermined fuzzy partitioning is required before generating fuzzy rules by constructing fuzzy membership functions automatically, because it decides which kind of partition will be used to construct fuzzy membership and rules automatically. A proper clustering technique can not only reduce the useless rule, but also can simplify the rule base to achieve the rule base optimization.

\section{THE STRUCTURE OF PROPOSED} MODEL - AN APPROXIMATED FOURPHASE FUZZY LEARNING

\section{ALGORITHM}

Basically, the purpose of the entire research anticipates constructing an automatic fuzzy inference system generation from data with the maximum performance and the minimum cost, and this fuzzy inference system can be decomposed into four main phases: pattern recognition, fuzzy inference engine, function approximation and system optimisation. In Phase 1: Pattern Recognition, the clustering algorithm tries to identify both the correct number of clusters and the appropriate locations for the corresponding clusters, and the purpose of this phase cannot only try to find out the optimal number of clusters but also try to avoid the problem of curse of dimensionality in advance. In Phase 2: Fuzzy Inference Engine, based on the corresponding clusters identified by the Phase 1 , the fuzzy membership function and rules will be 
Volume 9 Issue 6 June 2021

generated by fuzzy system identification or fuzzy granular system identification automatically. In Phase 3: Function Approximation, in case the resultant clusters of Phase 1 make a poor performance resulting in a high degree of dimensionality or lower accuracy with improper cost in Phase 2, the original result of Phase 1 would be improved by certain approximation algorithms in Phase 3. In Phase 4: System
Optimization, in order to attain higher accuracy, fewer fuzzy rules and parameters, maximum performance, and minimum cost, the focus of this phase is put on improving the entire system by fine-tuning certain system optimisation techniques. Hence, the Brief Structure of The Anticipated Claims can be illustrated as follows:

\section{Pattern Recognition}

1. Output Context Clustering

2. Decision Tree

3. Hierarchical Clustering (Single-Linkage Clustering)

4. Hybrid Clustering Method

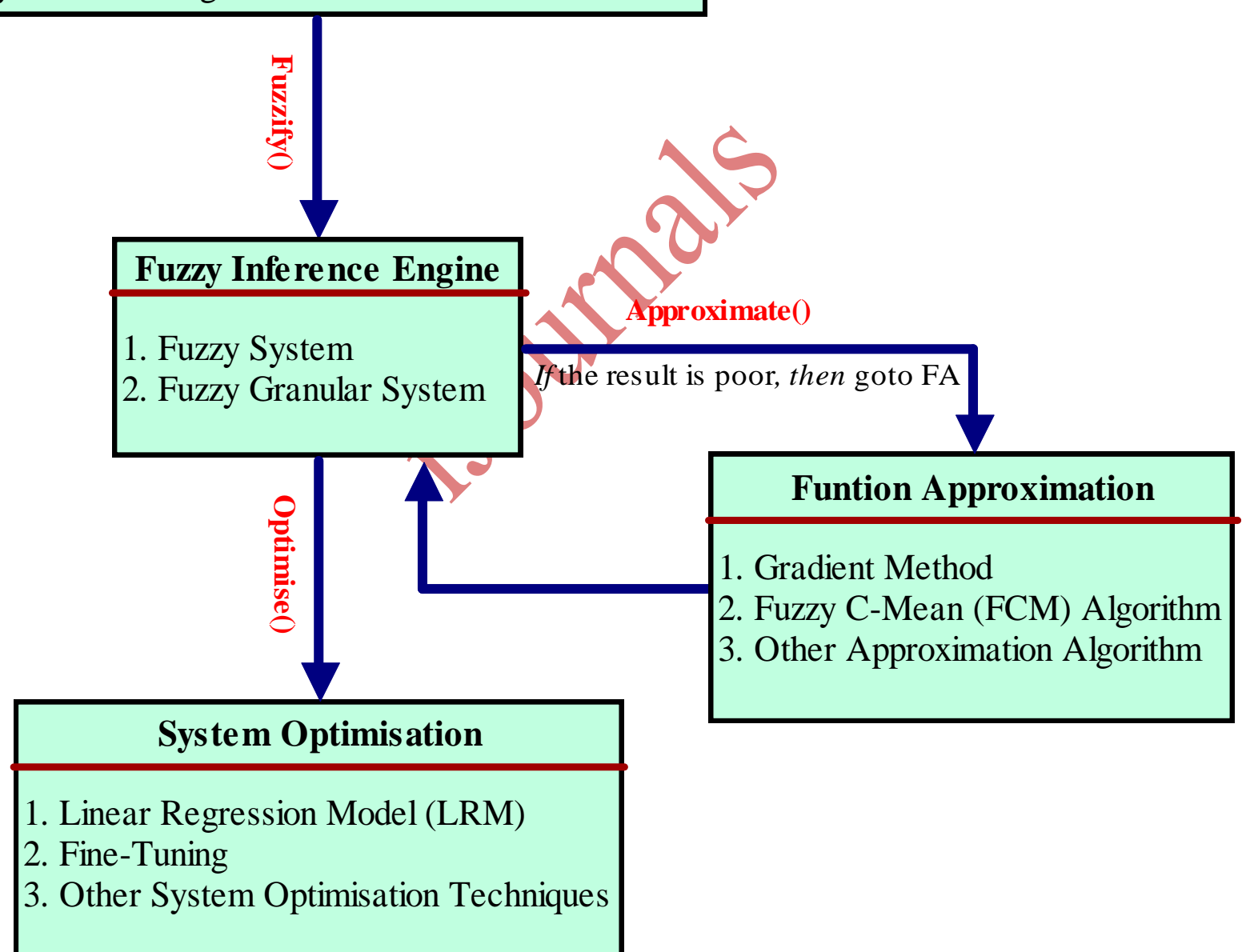

Fig. 1. The brief structure of the anticipated claims 


\section{DISCUSSION AND ANALYSIS}

For reaching the optimal achievement, this work tries to perform the advantages of the proposed methodology and improve the weaknesses of each method. Most important of all, how to achieve the best compromise between the accuracy of the approximation and the degree of the interpretability in the whole system by doing less effort and saving resources is the main purpose of this research. To sum up, this proposed system anticipates reaching the achievement of the following main features.

\section{(1) Low degree of the dimensionality}

In the aspect of low degree of the dimensionality, the clustering algorithms, such as output-context clustering, single-linkage clustering and decision tree algorithm, are the main methods trying to probably decrease the degree of the dimensionality by discovering approximately correct number of clusters and locating the discrete regions simultaneously. Also, the advantage will be expected to be demonstrated by comparing with other existing methods, such as classification and regression tree (CART), K-Means algorithm, or methods proposed by other papers.

(2) Highly accuracy of the approximation

In the aspect of high accuracy of the approximation, the approximation method, such as the gradient method, is being the main method trying to improve the accuracy by approximating the prototype if the accuracy of the original result cannot satisfy the requirement. Also, certain approximation method may be adopted if it has been developed with a better performance of the approximation.

\section{CONCLUSION}

This proposed system also aims to pursuit of reaching the achievement of the following sub-features if it's applicable:

(1) Well-understanding interpretability

In the aspect of well-understanding interpretability, the relevant fuzzy theories or certain neuro-fuzzy structure, such as fuzzy system, fuzzy granular system or ANFIS (Adaptive Neuro-Fuzzy Inference Systems), will be embedded whilst it can make a clearer legibility under certain conditions.

(2) Maximum performance and minimum cost

In the aspect of maximum performance and minimum cost, approaches of fine-tuning and techniques of system optimisation will be trying to get more performance by decreasing the cost.

However, it is difficult to develop an algorithm that is best in all aspects, so how to perform the main features and then balance the sub-features is still an increasing issue to the future work. 